\title{
A new validation method for clinical grade micro-encapsulation: quantitative high speed video analysis of alginate capsule
}

\author{
Ina Meiser • Sabine C. Müller • Friederike Ehrhart • \\ Stephen G. Shirley • Heiko Zimmermann
}

Received: 2 August 2013 / Accepted: 2 October 2013/Published online: 17 October 2013

(C) The Author(s) 2013. This article is published with open access at Springerlink.com

\begin{abstract}
Micro-encapsulation of cells or tissues is a promising treatment for hormone deficiency diseases (diabetes mellitus, hypoparathyroidism etc.). Alginate, a polymer from marine brown algae, is excellent for encapsulation. Its hydrated structure keeps out large antibodies and immune system cells while allowing diffusion of nutrients and therapeutic factors. Alginate droplets, containing cells, are crosslinked to form stable spherical hydrogel capsules. The position of grafts within the capsule, which decisively affects vulnerability to the host's immune response, has previously not been investigated during the process of crosslinking. We describe for the first time quantitative, high speed video analysis of two perpendicular projections of capsule shape and load during crosslinking. This gives characteristic "deformation plots" of the capsule and fully resolved "trajectories plots" of capsule load movement. We identify four stages of capsule formation, giving insights into physico-chemical processes involved. Further, we show that mechanical stress on the load can be deduced by measurement of the capsule's shape.
\end{abstract}

I. Meiser · S. C. Müller · F. Ehrhart · S. G. Shirley ·

H. Zimmermann

Division of Biophysics and Cryotechnology,

Fraunhofer Institute for Biomedical

Engineering IBMT, Ensheimer Str. 48,

66386 St. Ingbert, Germany

\section{H. Zimmermann $(\bowtie)$}

Department for Molecular and Cellular Biotechnology/

Nanotechnology, Saarland University, P.O. Box 151150,

66041 Saarbrücken, Germany

e-mail: heiko.zimmermann@ibmt.fraunhofer.de

\section{Introduction}

Today, immobilization and micro-encapsulation is used in biotechnological, chemical/pharmaceutical bulk production and also in regenerative medicine (Lanza et al. 1996; Zimmermann et al. 2007a, 2008). Encapsulated cells that release therapeutic factors are promising for the treatment of a variety of diseases (e.g. diabetes mellitus), since nonautologous cells and tissue can be transplanted without immunosuppressive therapies (Senior 2001; De Vos et al. 2006; Tao 2006; Zimmermann et al. 2007b; Skyler and Ricordi 2011). The cells are protected from the host's immune response by an artificial membrane or matrix, while nutrients and therapeutic factors can pass (Zimmermann et al. 2008). Furthermore cells behave in a more natural way, retaining more physiological functionality, if they have three dimensional environments. Chondrocytes, for example, produce cartilage typical collagen II only when they are cultured in three dimensional environments (Steinert et al. 2003). The increasing interest of biomedicine in cell-matrix constructs promotes the development of high throughput culture techniques (Tendulkar et al. 2012) and increases the interest in cryopreservation of these constructs for retrospective investigations, pooling and stock keeping (Malpique et al. 2010; Zhang et al. 2009). Also, immobilized cells are valuable model systems for tissue, e.g. to test effects of a three dimensional environment on mitosis, apoptosis or differentiation of stem cells (Weber et al. 2002). In all cases, the choice of extra-cellular matrix is crucial, since immunoisolation characteristics, permeability and mechanical stability are important. Here, ultra-high viscosity alginate (clinical grade, own production) is the biomaterial of choice because this hydrogel is successfully established in animal trials as long-term stable and it triggers no response from the 
immune system (Zimmermann et al. 2001, 2005; Nafea et al. 2011). Alginate obtained from Lessonia nigrescens and Lessonia trabeculata is used in equal parts because this mixture, with a G:M ratio (guluronic and mannuronic acid, the monomers of alginate which influence polymerization and syneresis behaviour) of 65:35 was found to be most successful in our pre-clinical studies (Storz et al. 2010).

Electrical, vibration and coaxial airflow methods can produce micro-droplets of alginate solution. But, however produced, these droplets finally fall into a polymerization solution, where polysaccharide branches are cross-linked (Wolters et al. 1992). Micro-encapsulation in alginate has been tested in animal trials (Schneider et al. 2005; Elliott et al. 2007; Tuch et al. 2011). But some features of the encapsulation process have not yet been evaluated. These include the effect of mechanical forces when the capsule impacts the polymerization bath and the predominant decentralized position of the load within the capsule.

We have established a method, using high speed video, to characterize the polymerization process of alginate droplets in the Sect. 3.1 as well as load behaviour in 3D inside a capsule during this process (Sect. 3.2) (Meiser et al. 2009). Here, we used macroscopic capsules $(2 \mathrm{~mm}$ diameter) in order to examine load's movement during the impact and polymerization process. As we will show, this method gives reproducible and exact information about both, the physical chemistry of the alginate polymerization process as well as the properties of the used biomaterial. This technique is non-invasive and allows conclusions about limitations of the alginate microcapsule formation processes; gives insights into load localization and gives hints for process improvement.

\section{Materials and methods}

\subsection{Production of alginate solution}

We used dried algae stipes from $L$. nigrescens and $L$. trabeculata (from the coast of Chile) as raw material for alginate production. Alginate was extracted according to (Zimmermann et al. 2005) and alginate solution was prepared by mixing dried alginate with isotonic $\mathrm{NaCl}$ (Sigma, Schelldorf, Germany) solution $(0.9 \%(w / v))$ and leaving overnight in a rotation device. Concentrations of the alginate solutions were adjusted to $0.1-0.7 \%(\mathrm{w} / \mathrm{v})$ and stained with phenol red (Sigma, Schelldorf, Germany) for an enhanced visibility (about $2 \mathrm{mg} / \mathrm{ml}$ ).

\subsection{Production of alginate load models and cell culture}

Microscale alginate beads $(400-500 \mu \mathrm{m})$ of $0.7 \%(\mathrm{w} / \mathrm{v})$ alginate solution ( $1: 1 \mathrm{~L}$. nigrescens and $L$. trabeculata $)$ produced by the crystal gun device (Zimmermann et al. 2003), serve as load models, since biological materials lack of sufficient contrast for high speed video. To improve contrast these micro beads were stained with alcian blue (Sigma, Schelldorf, Germany, see Fig. 1b frontal and mirror view). For trajectory plots the beads were encapsulated in 0.5 and $0.7 \%$ alginate solution $(1: 1 \mathrm{~L}$. nigrescens and L. trabeculata). Murine fibroblasts (L929, DSMZ, Braunschweig, Germany) serves as cell load and were cultivated according manufacturer's advice. For deformation method, $1 \mathrm{E}+06$ cells were mixed in $1 \mathrm{ml} 0.7 \%(\mathrm{w} / \mathrm{v})$ alginate solution (1:1 L. nigrescens and L. trabeculata).

\subsection{Experimental setup}

A syringe equipped with a thin cannula $(0.4 \mathrm{~mm})$ was fixed above a transparent bath tank filled with crosslinking solution $(20 \mathrm{mM} \mathrm{BaCl}, 115 \mathrm{mM} \mathrm{NaCl}, 5 \mathrm{mM}$ His, all Sigma, Schnelldorf, Germany), isotonic NaCl-solution or distilled water. Here, the syringe was fixed at $d=13.5 \mathrm{~cm}$ above the transparent vessel (see Fig. 1a for a schematic drawing of the setup). The tank contained a glass scale and an aluminum mirror (Thorlabs, Dachau, Germany) placed at a $45^{\circ}$ angle, both visible to a high speed camera. A second aluminum mirror (Thorlabs, Dachau, Germany) was positioned outside the bath at a $45^{\circ}$ angle to the glass scale and opposite to the internal mirror. Two light sources (Olympus ILP-1) beside the high speed camera, lit the front view of the glass scale and (via the outer mirror) the side view. The mirror system gives a single image containing front and side views and allows analysis of the three dimensional capsule. The high speed camera was a Speed Cam Visario LT400 (Weinberger, Erlangen, Germany) with its corresponding software Visart. We used a rate of 1,000 frames per second and extracted single picture frames out of the raw data produced by the high speed camera.

\subsection{Evaluation procedure}

For deformation plots, a capsule's broadest width was measured for each image and plotted against time [indicated in Fig. $1 \mathrm{~b}(t=20.5 \mathrm{~ms})]$.

The load's trajectory was also mapped (see Fig. 2 for a schematic overview and Fig. 1b lateral and mirror view for an exemplary image of the trajectory evaluation). Firstly, the capsule's 3D centroid determined as well as the frontal and the lateral positions of the load (using ImageJ software; NIH, USA). Next the load's position was transformed into a coordinate system with the capsule's centroid as origin (indicated as red cross in Figs. 2, 4). For each frame the frontal and lateral load position were measured and transformed into three-dimensional coordinates of the load's 


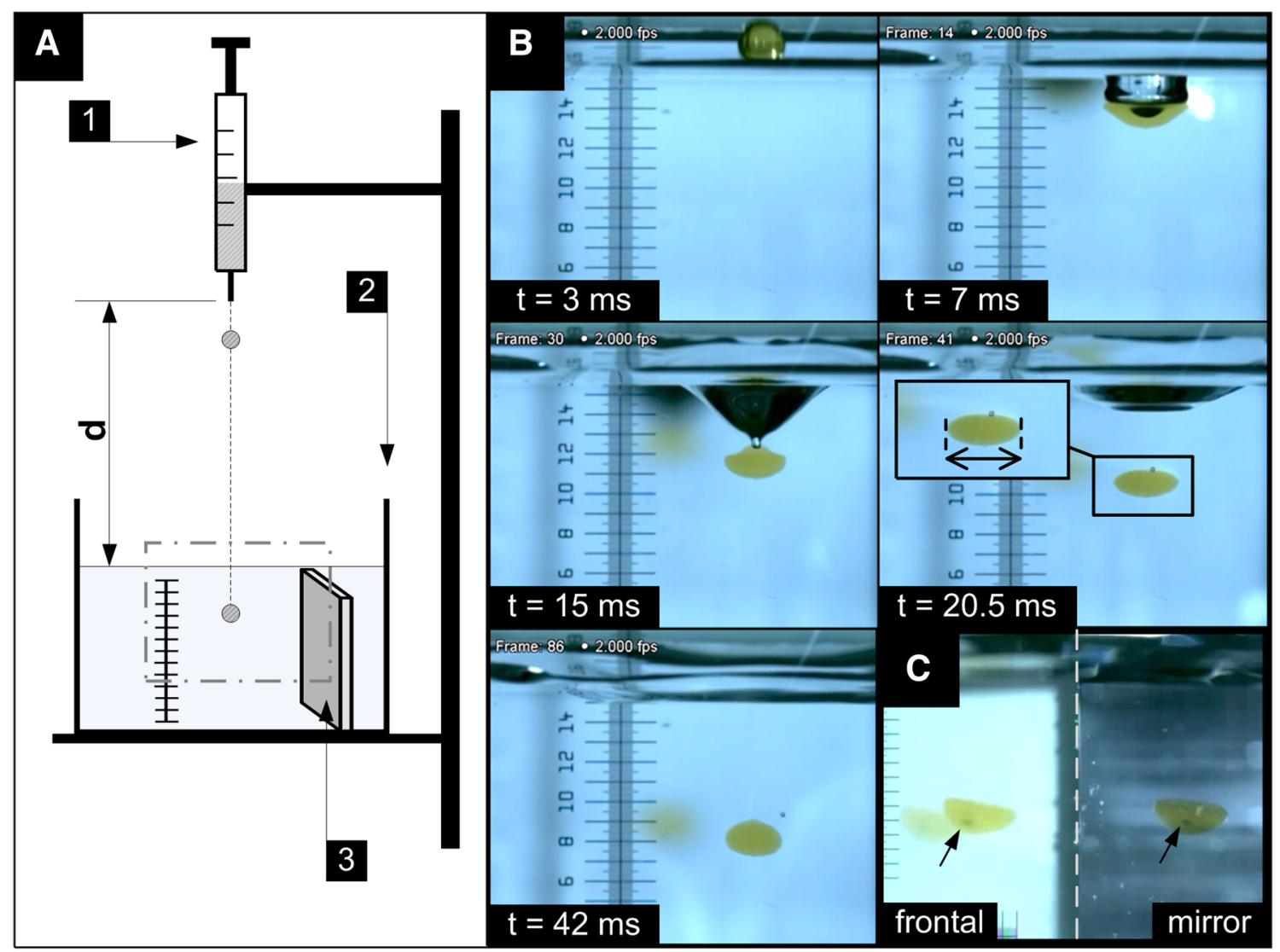

Fig. 1 a Setup schematic (frontal view): to generate droplets of similar size a syringe with blunt cannula (1) containing the sample solution was used. After falling a defined distance $d$, the droplet impacts in a vessel (2) containing polymerization or non-polymerization solution. The vessel itself is equipped with a scale bar and an internal mirror (3) providing a three-dimensional perspective. The scene is lit by two light sources at camera's position and an outer mirror assures illumination of the inner one (not shown). The dotted square indicates the area recorded by a high speed camera. b Example of resulting images in frontal view. Image at time $t=20.5 \mathrm{~ms}$ shows the evaluation procedure for deformations plots: a capsule's diameter at broadest width is measured for each image over a $120 \mathrm{~ms}$ time period. $\mathbf{c}$ Exemplary image of frontal and lateral view provided by the inner mirror
Fig. 2 The trajectory

evaluation procedure at time $t$. a Determination of an alginate capsule's (1) 3D centroid (3, marked as red cross) as well as the frontal and lateral graft model's position (2).

b Transformation of graft's model position into $Y X$ - and $Y Z$ coordinate systems in which the capsule's centroid serves as origin. c Resulting trajectory graph for one encapsulation run. One data point represents one position of a graft model inside the alginate capsule at a time $t$

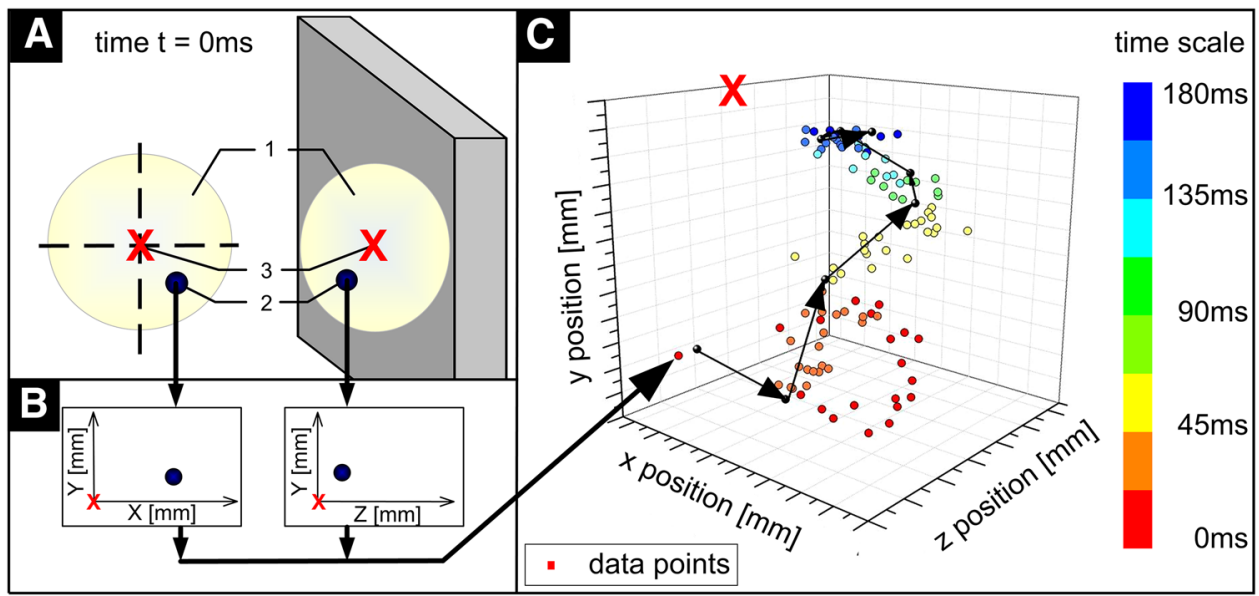

position. After plotting the positions a three-dimensional "Trajectory Graph" (see Fig. 2c) was obtained. The time was displayed as color-coded data points from red to blue (time interval $1 \mathrm{~ms}$ from frame 1 to $50,2 \mathrm{~ms}$ afterwards). A load's velocity $\vec{v}$ in a three dimensional capsule with load's coordinates $\mathrm{x}, \mathrm{y}, \mathrm{z}$ was calculated by: 

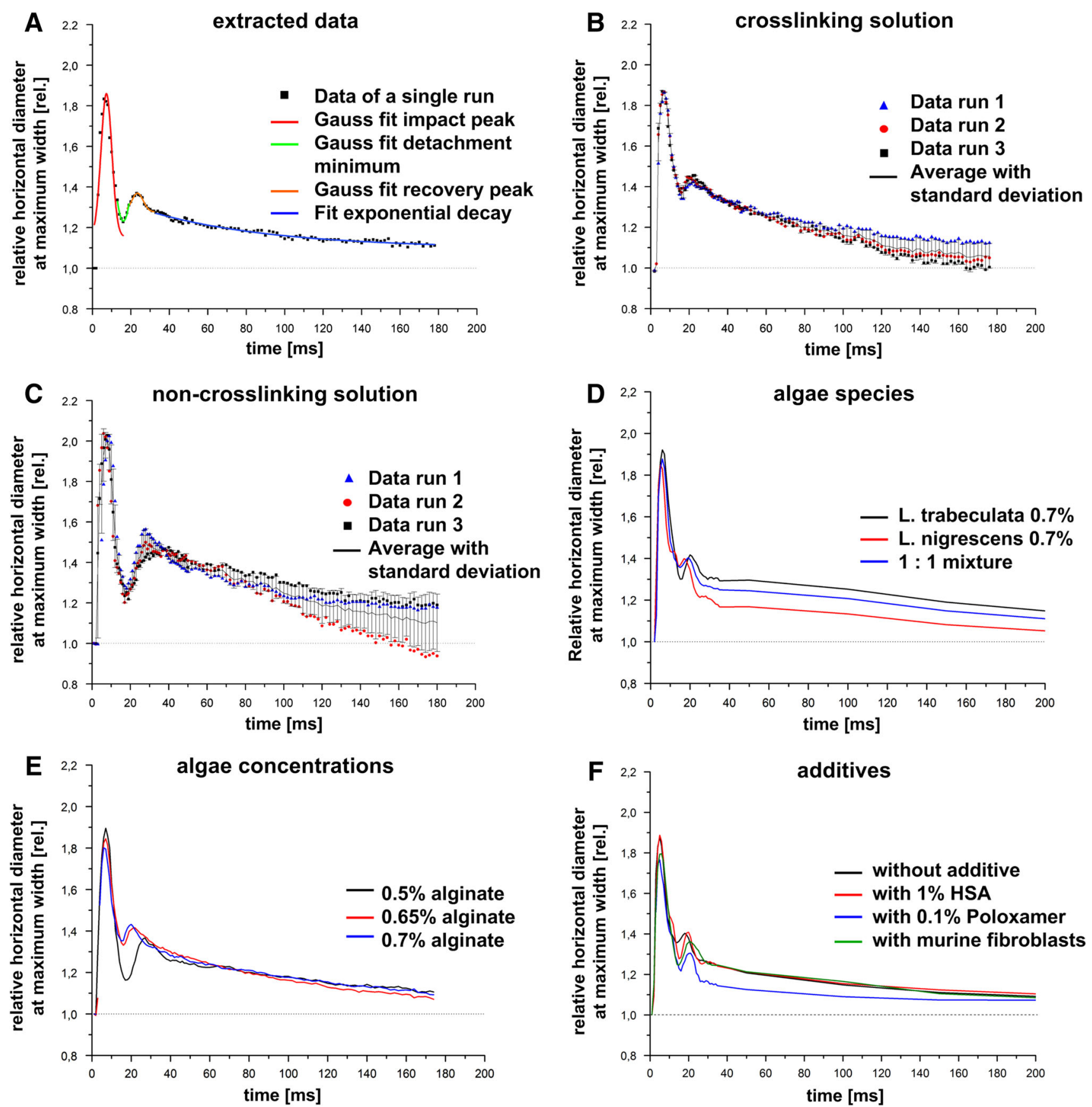

Fig. 3 Deformation plots of different experimental setups. a The diameter of an alginate droplet plotted against time gives the typical deformation plot that can be fitted by three Gauss curves and an exponential decay function. Deformation plots of $\mathbf{b}, \mathbf{c} 0.65 \%$ alginate droplets in crosslinking and non-crosslinking solution; d $0.7 \%$

alginate solutions of different origin: L. trabeculata, L. nigrescens and a 1:1 mixture of both; e Different alginate concentrations of 1:1 mixtures: $0.5,0.65$ and $0.7 \%$; f $0.7 \%$ alginate solutions with different additives: $1 \%$ Poloxamer, $1 \%$ HSA, murine fibroblasts and without supplements

$\vec{v}=\left(\begin{array}{c}v_{x} \\ v_{y} \\ v_{z}\end{array}\right)=\left(\begin{array}{c}d x / d t \\ d y / d t \\ d z / d t\end{array}\right)$ and $\overrightarrow{|v|}=\sqrt{v_{x}^{2}+v_{y}^{2}+v_{z}^{2}}$

The acceleration of a load inside a capsule therefore was calculated by:

$$
\begin{aligned}
\vec{a} & =\left(\begin{array}{l}
a_{x} \\
a_{y} \\
a_{z}
\end{array}\right)=\left(\begin{array}{l}
d^{2} x / d t^{2} \\
d^{2} y / d t^{2} \\
d^{2} z / d t^{2}
\end{array}\right)=\dot{\vec{v}} \text { and } \overrightarrow{|a|} \\
& =\sqrt{a_{x}^{2}+a_{y}^{2}+a_{z}^{2}}
\end{aligned}
$$




\section{Results}

\subsection{Deformation method}

\subsubsection{Characteristic stages during impinging process}

Observed from above, the impact of alginate droplets on polymerization solution looks like the impact of water droplet (data not shown). Observation from the side shows a very complex yet highly reproducible behaviour of the alginate capsules (Figs. 1b, 3), which can be divided into four different stages: At first, the alginate droplet is heavily deformed through the impact on the fluid surface (Fig. 1b time $t=7 \mathrm{~ms}$; impact stage) and pressed under water (Fig. $1 \mathrm{~b} t=7 \mathrm{~ms}$ and $t=15 \mathrm{~ms}$ detachment stage) due to its kinetic energy. After detachment of the alginate from the fluid surface the droplet takes the shape of an erythrocyte (Fig. 1b $t=20.5 \mathrm{~ms}$, recovery stage) and sinks slowly to the bottom of the vessel while reforming a spherical shape (Fig. $1 \mathrm{~b} t=42 \mathrm{~ms}$, decay stage).

\subsubsection{Effect of ions in bath tank solution}

Characteristic deformation plots (for different alginate and impact conditions) were formed by plotting the capsule's maximum width against time (Fig. 3a). The impact peak (when the droplet hits the surface) contains the maximal deformation. At the detachment minimum the droplet detaches from the fluid surface which is accompanied by horizontal size reduction. While sinking to the bottom, the alginate droplet increases its horizontal size quickly to the recovery peak and then slowly changes toward a spherical shape (which can be found in the deformation plot as exponential decay). The sequence of deformation steps was highly reproducible for any given crosslinking solution as shown by performance of several experiments (Fig. 3b).

If the polymerization $\mathrm{BaCl}_{2}$-solution was exchanged for ultra pure water there were obvious differences in the alginate deformation plot (Fig. 3c). The initial impact peak is larger, whereas horizontal size at the detachment minimum is smaller implying stronger deformation of the alginate bead. Furthermore, the bead takes no spherical shape but sinks to the bottom and starts to dissolve.

\subsubsection{Effect of algae composition and concentration}

For biomedical purposes alginate with a guluronic $(\mathrm{G})$ to mannuronic (M) acid ratio of 65:35 shows appropriate crosslinking properties for viscosity and pore sizes. This ratio corresponds to a 1:1 mixture of Lessonia nigrescens and Lessonia trabeculata alginate. Each line in Fig. 3d shows the mean value of 5 runs. The deformation plot shows that the mixture line is located between the pure species, whereas pure high-M Lessonia nigrescens alginate seems to be less deformable than pure high-G Lessonia trabeculata alginate. In varying concentration of alginate, solutions of $0.3,0.5,0.65$ or $0.7 \%(w / v)$ were used. The $0.3 \%(\mathrm{w} / \mathrm{v})$ solution could not be used for measurements because the alginate remained at the surface, where due to optical effects of the surface at the edges of the vessel the size could not be measured. Figure $3 e$ shows the deformation plots of different concentration of alginate. Obviously the deformation of an alginate capsule was higher the low alginate concentration (lower viscosity). Impact velocity, modified through height of fall and thus kinetic energy, modifies impact deformation. Former work shows that the higher the starting point of the alginate capsule the more deformation occurs (Meiser et al. 2007): For $0.65 \%$ alginate solution in crosslinking solution, the relative horizontal diameter spans from 1.62 for $d=6.75 \mathrm{~cm}$ height of fall through 1.81 for $13.5 \mathrm{~cm}$ to 2.02 for a $27 \mathrm{~cm}$ height.

\subsubsection{Effect of additives}

Some additives, interesting for micro-encapsulation of therapeutically relevant cells, are found which influences the viscosity of alginate solutions. Addition of $1 \%(\mathrm{w} / \mathrm{v})$ HSA (human serum albumin) does not increase the impact deformation but lowers the detachment minimum while showing the same recovery peak and exponential decay as pure alginate solution (Fig. 3f). After adding $0.1 \%$ Poloxamer, a polymer improving shear stress tolerance in cells (Ramirez and Mutharasan 1990; al-Rubeai et al. 1993), the impact peak is decreased as well as detachment minimum and recovery peak. During exponential decay, alginate capsules containing Poloxamer reach their final diameter faster.

In further experiments, the influence capsule's load on the deformation was examined. Murine fibroblasts in alginate solution influence the plot by decreasing the impact deformation and delaying the recovery peak in comparison to the same alginate solution without cells, yet single cells cannot be seen in the current setup in order to apply the trajectory methods (Fig. 3f).

\subsection{Trajectory method}

\subsubsection{Trajectory plots}

For high resolution in a three dimensional high speed video analysis, micro alginate beads, stained with alcian blue were used for a sufficient contrast in both, frontal and lateral view (see Fig. 1b). To detect significant behaviour and load movements inside a capsule during the polymerization processes, 9 experiment runs with $0.5 \%$ alginate solution and 6 with $0.7 \%$ alginate solution for the 3D 
Trajectory Method were performed. In particular, each encapsulation run was analyzed as described in methods (see Fig. 2). The force $F$ of a spherical particle with radius $r$ moving through a liquid of viscosity $\mu$ with a velocity $v$ is describes by Stoke's equation:

$F=6 \cdot \pi \cdot \mu \cdot r \cdot v$

Given that the particle mass $m$ for radius $r$ and density $\rho$ is:

$m=4 \cdot \pi \cdot r^{3} \cdot \frac{\rho}{3}$

Acceleration of a particle is:

$a=\frac{d v}{d t}=\frac{-3 \cdot 6 \pi \mu r v}{4 \pi r^{3} \rho}=\frac{-v \cdot 9 \mu}{2 \rho r^{2}}$

What integrates to:

$v=v_{0} \cdot \exp -\left(\frac{t}{\frac{2 \rho r^{2}}{9 \mu}}\right)$

So, the stopping time $t$ and the stopping distance $d$ of a particle is:

$t=\frac{2 \rho r^{2}}{9 \mu}$ and $d=\frac{v_{0} 2 \rho r^{2}}{9 \mu}$

To enable a comparison of all results, each Trajectory Graph is normalized to equal axes and the origin (centroid) is marked as red cross. Figure 4a shows an overlay of all 15 trajectory graphs and exhibits as expected that the maximal expansion of the bead's trajectory depends on the alginate concentration. Graft models encapsulated in $0.5 \%$ alginate (lower viscosity) reveal greater distances between particular trajectory positions than in higher concentrations. For the lower viscous $0.5 \%$ alginate solution absolute distances of $0.79 \pm 0.46 \mathrm{~mm}$ between start and end point of a graft during an encapsulation run are observed. Load models in a $0.7 \%$ concentrated alginate solution display only a distance mean value of $0.25 \pm 0.09 \mathrm{~mm}$. Considering the overlay of all 15 Trajectory Graphs in a density plot (see Fig. 4b) a "NoGo Area" of a capsule's load is clearly brought out: No load model ever reaches this area inside a droplet during capsule formation. The area encloses the region around the centre and in a V-shape above the centre in the direction of the Y-axis. Most loads in $0.5 \%$ alginate capsules and all loads in $0.7 \%$ alginate capsules are located in the lower half.

\subsubsection{Coherence plots}

We calculated the velocity and acceleration of loads within a capsule (for 0.5 and $0.7 \%$ alginate each, $\mathrm{N}=6$ ) and show that velocity and acceleration are coherent with the horizontal deformation (see Fig. 5). As shown in Fig. 5, highest velocity and acceleration of a load is detected at the beginning of the capsule formation process during the impact stage due to little alginate cross linking. In $0.5 \%$ alginate load velocity reaches $0.79 \pm 0.1 \mathrm{~mm} / \mathrm{ms}$, in $0.7 \%$ alginate $0.75 \pm 0.09 \mathrm{~mm} / \mathrm{ms}$. During decay phase velocity and acceleration show damped oscillation characteristics, mean velocity in this phase is $0.13 \pm 0.02 \mathrm{~mm} / \mathrm{ms}$ for $0.5 \%$ alginate and $0.14 \pm 0.02 \mathrm{~mm} / \mathrm{ms}$ for $0.7 \%$ alginate. Acceleration of particles in both alginate concentrations is nearly the same: mean value for both concentrations in impact phase is $0.07 \pm 0.02$ and $0.03 \pm 0.01 \mathrm{~mm} / \mathrm{ms}^{2}$ for the overall impinging process.

\section{Discussion}

Despite the fact that drop impacts have been studied for over a century now, a detailed understanding is still lacking (Engel 1955; Fedorchenko and Wang 2004; Worthington 1908). Most studies examine fluid to fluid or fluid or solid impacts and the effects occurring on the surface hit by the drops (Jung and Hutchings 2012; Kang and Lee 2000; Lohse et al. 2004; Manzello and Yang 2002; Mutchler and Hansen 1970; Scheller and Bousfield 1995; Xu et al. 2005). However, the impact of alginate in polymerization solution is a special case in impact research and analyzing surface effects does not map the whole process. Alginate solution is a non-newtonian fluid that undergoes a change in viscosity after contact with crosslinking solution (Siew et al. 2005; Storz et al. 2010; Becker and Kipke 2002) and, to our knowledge, there is no system published which describes in detail the behaviour of the impinging droplet and the change of viscosity during impact. Observing the behaviour of the polymerization solution from above a kind of crown building occurs, which is typical for the impact into liquids or even loose sand (Lohse et al. 2004). Also the form of the impact crater and the jet formation is qualitatively the same. Considering only the alginate capsule, we showed there is a complex and reproducible behaviour which can be divided in four characteristic stages: (1) deformation, (2) detachment, (3) recovery and (4) decay to sphere, comparable to investigations on an impact on a solid surface described in (Rioboo et al. 2001) and (Jung and Hutchings 2012). In the case of alginate capsules in crosslinking solution, air bubble entrapment (as reported by Mehdi-Nejad et al. 2003; Mohamed-Kassim and Longmire 2003) occurred very infrequently and was not considered in our examinations since accurate graft position assignments were not possible. This new observation and evaluation method reduces a $3 \mathrm{D}$ system to $2 \mathrm{D}$ pictures for a definite synchronization and feasible data handling. Rotation symmetry was proven by a multi mirror system, providing 


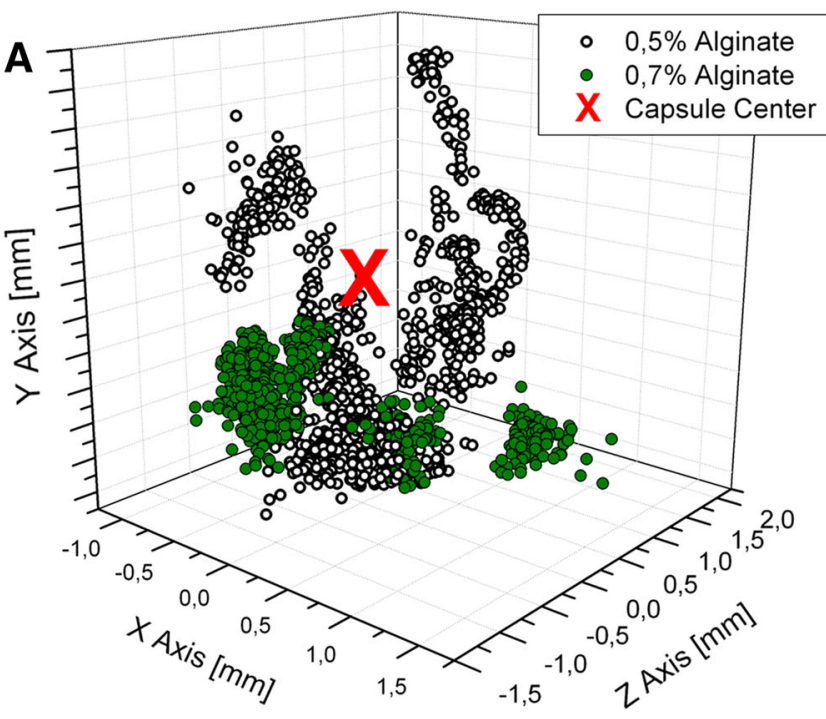

Fig. 4 a Trajectory graph overlay of 15 encapsulation runs. Data points are colored corresponding to alginate concentrations. Centroid is marked as red cross. Capsules of $0.5 \%$ alginate ( 9 trajectories shown in white bullets) show bigger absolute movements $(0.79 \pm 0.46 \mathrm{~mm})$

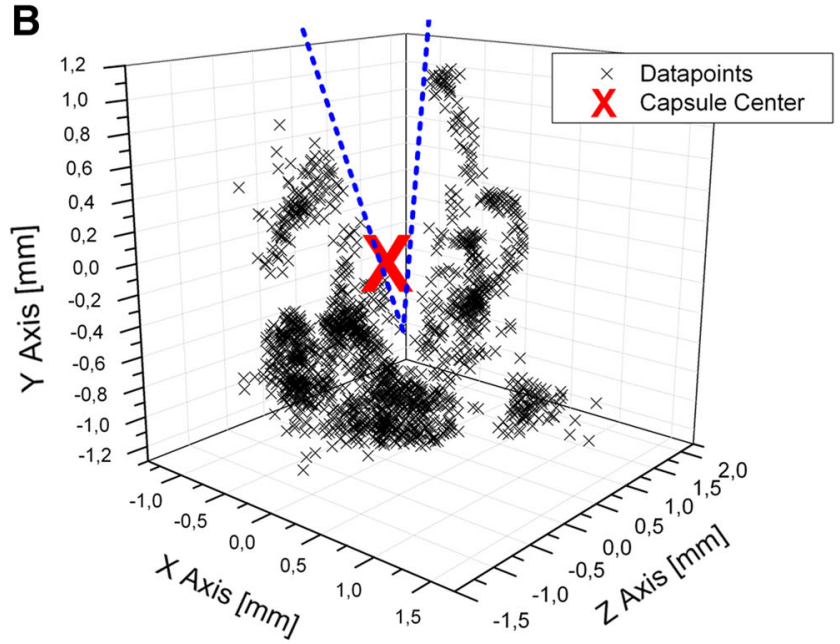

than capsules of $0.7 \%$ alginate $(0.25 \pm 0.09 \mathrm{~mm})$ shown in green. b Density plot of all encapsulation runs $(N=15)$ shows the No-Go area (dashed blue line) and that most loads are located in the lower half of the capsules

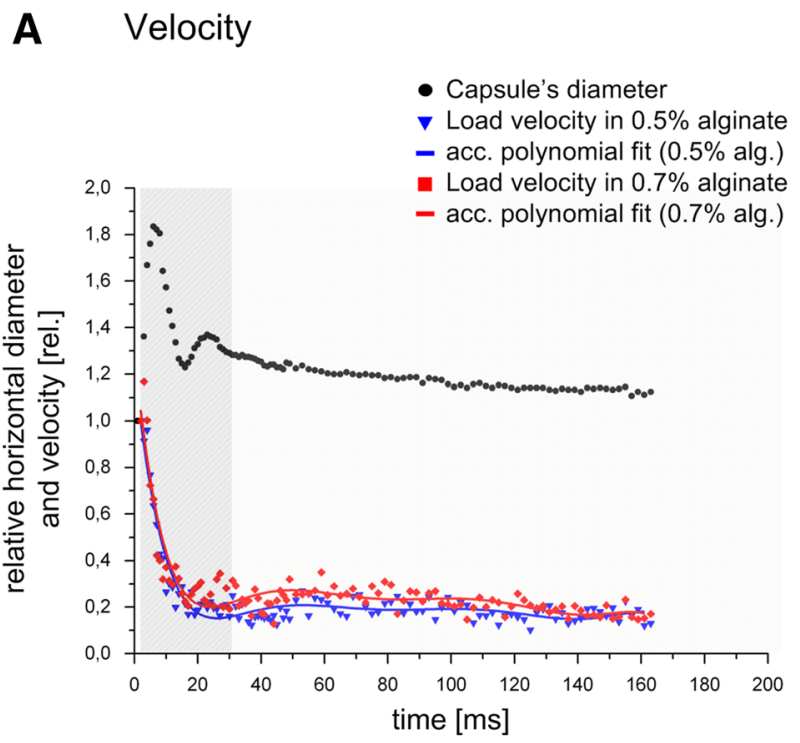

Fig. 5 a Coherence plot of load's velocity in different alginate concentrations ( $N=6$ for each concentration). An exemplary deformation plot (black dot data) is given as reference (see also Fig. 3a). Impact, Detachment and Recovery stage of capsule's deformation is highlighted as grey plain. For velocity, mean values are shown as data

three dimensional views of the process and also enabling us to follow graft's trajectories inside capsules.

We found that plotting horizontal diameter versus time was a precise method to detect fine differences in deformation behaviour of alginate droplets with different compositions and additives during polymerization. The resulting plot resembles a damped oscillatory wave. A disadvantage is the distortion of the pictures at the water-

\section{B Acceleration}

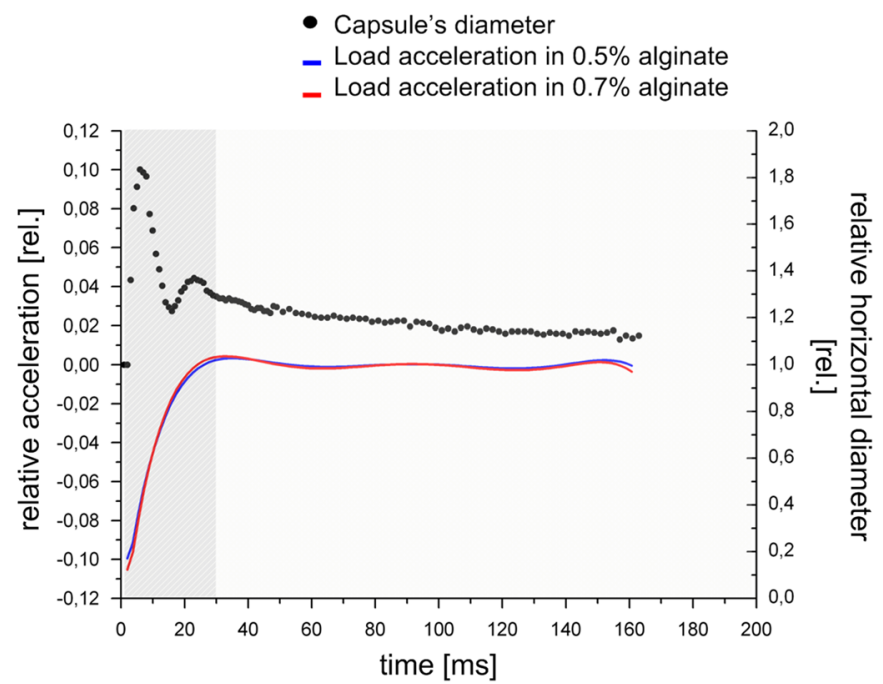

points (standard deviations are subdivided). Each data set is polynomial fitted and displayed as blue ( $0.5 \%$ alginate) or red $(0.7 \%$ alginate $)$ line. b Coherence plot of load's acceleration: acceleration is the derivation of the velocities polynomial fit

air interface and it is not easy, sometimes not possible, to measure the size of the alginate capsule or to detect the load's position due to glare. Furthermore, staining of the alginate capsule is necessary to have sufficient contrast with the surrounding liquid, which has unknown consequences on the alginates' deformation behaviour. The use of an oversized model system (syringe, which produces $2 \mathrm{~mm}$ capsules, rather than the original encapsulation 
machine was experimentally necessary for the trajectory method in order to determine the load's position, because the droplets produced by the machine are too small to be measured optically with adequate resolution. Although the deformation stages can be observed in miniature as well, the method does not have the resolution for tracking the load at this scale (data not shown).

The extent of deformation that a droplet experiences while impacting a liquid is determined by the following factors: droplet diameter; viscosity, concentration, load and composition of the alginate solution, impact velocity (determined by gravity and height) and surface tension of the solution. Various dimensionless groups have been defined, e.g. Reynolds number, Froude number and, most importantly, Weber number. According to these it can be predicted, e.g. if the droplet coalesces or splashes as soon as it hits the liquid surface (Worthington 1908). At low Weber numbers (slow impact velocity) the droplet coalesces. Beginning at a critical Weber number jet formation occurs and the shape of the jet changes from short and broad to high and thin with further increase. Fragmentation of the jet into secondary droplets occurs with high Weber numbers but this was not observed with alginate. Basic problem for the diffusive polymerization process here is that different dimensionless groups govern for the different stages and a scaling of the whole is not possible (Weber number for impact, Reynolds number for particle distortion and Froude number for gravitational effects). With alginate, the impact deformation was influenced by polymerization ions and the content of guluronic- and mannuronicacid. Alginate itself is a biopolymer and therefore inhomogeneous across different algae collection and extraction batches. Other authors have even reported different cellular behaviour in various alginate compositions (De Vos et al. 1997; Kulseng et al. 1999). Differences in the deformation of alginate droplets impacting polymerizing or non-polymerizing solutions are obvious (see Fig. 3b, c). Differences occur in the first 3-5 ms after contact at the impact maximum. Deformation is larger in non-polymerizing solutions, which implies that polymerization starts immediately and reduces broadening of the capsules after impact. The difference between more G-rich and M-rich alginate in deformation plots shown in Fig. 3d could stem from the higher viscosity of $\mathrm{M}$-alginate or different crosslinking properties. In general, high-viscosity alginates cross-linked with $\mathrm{Ba}^{2+}$ show different properties to low-viscosity alginates crosslinked with $\mathrm{Ca}^{2+}$. Current production of immune-isolating capsules is by impact polymerization, which mechanically stresses graft cells and tissues (e.g. Langerhans islets). Shear stress or friction can alter morphology (cytoskeleton) and physiology of cells and this can have adverse affects on cell functionality (Papoutsakis 1991). Stress can be reduced in this system by reducing impact velocity (height of fall) or by increasing viscosity. In bioreactor engineering, shear stress is a key parameter and higher viscosity reduces stress in turbulent animal cell culture (Croughan et al. 1989; Lakhotia and Papoutsakis 1992). However, these two parameters reach soon their limits. Ultra-high viscosity alginate cannot be dissolved in concentrations higher than $1 \%(\mathrm{w} / \mathrm{v})$ (and nutrient diffusion is reduced at high alginate lattice densities). For Langerhans' islet an alginate concentration of $0.7 \%$ is optimal (or $0.65 \%$ when crosslinked with the crystal gun; Zimmermann et al. 2003). The coaxial air flow needed to produce alginate capsules of small size $(200-800 \mu \mathrm{m})$ accelerates the droplets and a certain height of fall may be necessary to slow them down subsequently. The addition of HSA increases the viscosity of alginate whereas the addition of poloxamer does not (at $0.1 \%$ ). The deformation plot, however, is able to show fine and complex differences during the impact. Considering Fig. $3 \mathrm{~d}$, e and $\mathrm{f}$ it is obvious that the differences between the single deformation plots cannot be reduced to mere viscosity changes: The final diameters of alginate beads (after $200 \mathrm{~ms}$ observation time) of different algae sources in Fig. $3 \mathrm{~d}$ are different while the impact peak, detachment minimum and recovery peak are almost the same. Using different alginate concentrations, the detachment minimum and recovery peak vary while the exponential decay is similar. HSA and poloxamer improve survival of encapsulated Langerhans' islets but only poloxamer decreases impact peak, detachment minimum and, drastically, the exponential decay. The final diameter after $200 \mathrm{~ms}$ is not affected by additives but is influenced by algae source (L. nigrescens or L. trabeculata or mix). This could reflect changes in polymerization kinetics due to binding site availability, changing diffusion coefficient or surface tension. The shifts in deformation plots for encapsulated murine fibroblasts show that empty capsules can be identified with this method.

The location of the load inside a capsule is crucial for success of transplantation therapies (de Groot et al. 2004) since a peripheral position may trigger an immune response from the host. To our knowledge, no previous approach offers a non-invasive analysis of loads in three-dimensional space similar to trajectory analysis. In particular, the "NoGo Area" may have significant effects on biology; within this area the probability of finding a load is very low. This, and the fact that particles mostly stay in the lower half of a capsule, as displayed in Fig. 4b, is inertia. The larger displacement of particles in low viscous alginate shown in Fig. $4 \mathrm{a}$ occurs most probably to following reasons: According to Stokes' function, particles with equal inertia inside a falling capsule experience less frictional force in capsules of low viscosity. This fact enlarges their stopping time in an impinging alginate capsule, what presses the particles at rim positions. Also, low viscosity alginate 
capsules spread wider during the impact than high viscous ones (see Fig. 3e), what again reduced the distance of a particle to the capsule's rim. Furthermore, crosslinking procedure starts as soon as the alginate capsule submerges and since there are fewer linking sites in less concentrated alginate solution, diffusion and thus crosslinking occurs faster. Particles in rim positions are embedded earlier since crosslinking occurs there first. Thus they are forced to move slower and experience different displacements relative to the centroid than particles that are located closer to the middle. The difference in velocity is displayed in the coherence plot in Fig. 5a and the different displacements of particles are displayed in Fig. 4a. In Fig. 3e the faster crosslinking process of lower concentrated alginate is visible in the delayed and smaller recovery peak. Based on these results and the ability to analyze particle positions by the trajectory plot, less promising transplant candidates can be rejected. The viability of encapsulated cells may be improved by refining the impact process by monitoring with the Deformation and Trajectory Method. Harmful effects on loads due to acceleration and shear forces can be estimated and possibly minimized. Also "No-Go-Areas" can be identified for encapsulation technologies and help to avoid them in order to enable a more central graft position. First experiments show that a computer aided automation of these evaluation procedures is possible (data not shown). This could lead to a routine on-line analysis and optimisation tool in alginate capsule production. Using Deformation and Trajectory Plots based on high speed videos obtained from alginate droplet impact will lead to more detailed information about the physico-chemical properties of alginate solutions. Furthermore, these methods are feasible for observing polymerization kinetics with a sophisticated precise synchronized mirror setup. We have been able to investigate polymerization processes non-invasively and provide methods for batch screening of capsule formation as well as for load behaviour inside alginate capsules. These new techniques enable improved productions of clinically relevant hydrogel-based transplants.

Open Access This article is distributed under the terms of the Creative Commons Attribution License which permits any use, distribution, and reproduction in any medium, provided the original author(s) and the source are credited.

\section{References}

al-Rubeai M, Emery AN, Chalder S, Goldman MH (1993) A flow cytometric study of hydrodynamic damage to mammalian cells. J Biotechnol 31(2):161-177

Becker TA, Kipke DR (2002) Flow properties of liquid calcium alginate polymer injected through medical microcatheters for endovascular embolization. J Biomed Mater Res 61(4):533-540. doi:10.1002/jbm.10202
Croughan MS, Sayre ES, Wang DI (1989) Viscous reduction of turbulent damage in animal cell culture. Biotechnol Bioeng 33(7):862-872. doi:10.1002/bit.260330710

de Groot M, Schuurs TA, van Schilfgaarde R (2004) Causes of limited survival of microencapsulated pancreatic islet grafts. J Surg Res 121(1):141-150. doi:10.1016/j.jss.2004.02.018

De Vos P, De Haan B, Van Schilfgaarde R (1997) Effect of the alginate composition on the biocompatibility of alginate-polylysine microcapsules. Biomaterials 18(3):273-278 pii:S0142961296001354

De Vos P, Faas MM, Strand B, Calafiore R (2006) Alginate-based microcapsules for immunoisolation of pancreatic islets. Biomaterials 27(32):5603-5617 pii:S0142-9612(06)00600-4

Elliott RB, Escobar L, Tan PL, Muzina M, Zwain S, Buchanan C (2007) Live encapsulated porcine islets from a type 1 diabetic patient $9.5 \mathrm{yr}$ after xenotransplantation. Xenotransplantation 14(2):157-161. doi:10.1111/j.1399-3089.2007.00384.x

Engel OG (1955) Water drop collisions with solid surfaces. J Res Natl Bur Stand 54(5):281-298

Fedorchenko AI, Wang AB (2004) On some common features of drop impact on liquid surfaces. Phys Fluids 16(5):1349-1365

Jung S, Hutchings IM (2012) The impact and spreading of a small liquid drop on a non-porous substrate over an extended time scale. Soft Matter 8:2686-2696

Kang BS, Lee DH (2000) On the dynamic behavior of a liquid droplet impacting upon an inclined heated surface. Exp Fluids 29(4):380-387

Kulseng B, Skjak-Braek G, Ryan L, Andersson A, King A, Faxvaag A, Espevik T (1999) Transplantation of alginate microcapsules: generation of antibodies against alginates and encapsulated porcine islet-like cell clusters. Transplantation 67(7):978-984

Lakhotia S, Papoutsakis ET (1992) Agitation induced cell injury in microcarrier cultures. Protective effect of viscosity is agitation intensity dependent: experiments and modeling. Biotechnol Bioeng 39(1):95-107. doi:10.1002/bit.260390114

Lanza RP, Hayes JL, Chick WL (1996) Encapsulated cell technology. Nat Biotechnol 14(9):1107-1111. doi:10.1038/nbt0996-1107

Lohse D, Bergmann R, Mikkelsen R, Zeilstra C, van der Meer D, Versluis M, van der Weele K, van der Hoef M, Kuipers H (2004) Impact on soft sand: void collapse and jet formation. Phys Rev Lett 93(19):198003

Malpique R, Osorio LM, Ferreira DS, Ehrhart F, Brito C, Zimmermann H, Alves PM (2010) Alginate encapsulation as a novel strategy for the cryopreservation of neurospheres. Tissue Eng Part C Methods 16(5):965-977. doi:10.1089/ten.TEC.2009.0660

Manzello SL, Yang JC (2002) An experimental study of a water droplet impinging on a liquid surface. Exp Fluids 32:580-589. doi:10.1007/s00348-001-0401-8

Mehdi-Nejad V, Mostaghimi J, Chandra S (2003) Air bubble entrapment under an impacting droplet. Phys Fluids 15(1):173-183

Meiser I, Shirley SG, Zimmermann U, Zimmermann H, Ehrhart F (2007) Quantitative High Speed Video Analysis to embeddingimmobilization with alginates. 12 Leipziger Workshop 2007 Poster Session

Meiser I, Müller SC, Gepp MM, Zimmermann H, Ehrhart F (2009) Quantitative high speed video analysis of biopolymer encapsulated cells while capsule formation World Congress on Medical Physics and Biomedical Engineering, September 7-12, 2009, Munich, Germany. IFMBE Proc 22(17):2255-2258

Mohamed-Kassim Z, Longmire EK (2003) Drop impact on a liquidliquid interface. Phys Fluids 15(11):3263-3273

Mutchler CK, Hansen LM (1970) Splash of a waterdrop at terminal velocity. Science 169(3952):1311-1312. doi:10.1126/science. 169.3952 .1311

Nafea EH, Marson A, Poole-Warren LA, Martens PJ (2011) Immunoisolating semi-permeable membranes for cell 
encapsulation: focus on hydrogels. $\mathrm{J}$ Control Release 154(2):110-122 pii:S0168-3659(11)00237-9

Papoutsakis ET (1991) Fluid-mechanical damage of animal cells in bioreactors. Trends Biotechnol 9:427-437

Ramirez OT, Mutharasan R (1990) The role of the plasma membrane fluidity on the shear sensitivity of hybridomas grown under hydrodynamic stress. Biotechnol Bioeng 36(9):911-920. doi:10. 1002/bit.260360906

Rioboo R, Tropea C, Marengo M (2001) Outcomes from a drop impact on solid surface. Atomization Sprays 11(2):155-165

Scheller BL, Bousfield DW (1995) Newtonian drop impact with a solid surface. AIChE J 21(6):1357-1367. doi:10.1002/aic. 690410602

Schneider S, Feilen PJ, Brunnenmeier F, Minnemann T, Zimmermann $\mathrm{H}$, Zimmermann U, Weber MM (2005) Long-term graft function of adult rat and human islets encapsulated in novel alginate-based microcapsules after transplantation in immunocompetent diabetic mice. Diabetes 54(3):687-693. doi:10.2337/diabetes.54.3.687

Senior K (2001) Encapsulated cell technology provides new treatment options. Drug Discov Today 6(1):6-7 pii:S1359-6446(00)01620-2

Siew CK, Williams PA, Young NW (2005) New insights into the mechanism of gelation of alginate and pectin: charge annihilation and reversal mechanism. Biomacromolecules 6(2):963-969. doi:10.1021/bm0493411

Skyler JS, Ricordi C (2011) Stopping type 1 diabetes: attempts to prevent or cure type 1 diabetes in man. Diabetes 60(1):1-8. doi:10.2337/db10-1114

Steinert A, Weber M, Dimmler A, Julius C, Schutze N, Noth U, Cramer H, Eulert J, Zimmermann U, Hendrich C (2003) Chondrogenic differentiation of mesenchymal progenitor cells encapsulated in ultrahigh-viscosity alginate. J Orthop Res 21(6):1090-1097. doi:10.1016/S0736-0266(03)00100-1

Storz H, Zimmermann U, Zimmermann H, Kulicke WM (2010) Viscoelastic properties of ultra-high viscosity alginates. Rheol Acta 49:155-167. doi:10.1007/s00397-009-0400-x

Tao W (2006) Application of encapsulated cell technology for retinal degenerative diseases. Expert Opin Biol Ther 6(7):717-726. doi:10.1517/14712598.6.7.717

Tendulkar S, Mirmalek-Sani SH, Childers C, Saul J, Opara EC, Ramasubramanian MK (2012) A three-dimensional microfluidic approach to scaling up microencapsulation of cells. Biomed Microdevices 14(3):461-469. doi:10.1007/s10544-011-9623-6

Tuch BE, Hughes TC, Evans MD (2011) Encapsulated pancreatic progenitors derived from human embryonic stem cells as a therapy for insulin-dependent diabetes. Diabetes Metab Res Rev 27(8):928-932. doi:10.1002/dmrr.1274

Weber M, Steinert A, Jork A, Dimmler A, Thurmer F, Schutze N, Hendrich C, Zimmerman U (2002) Formation of cartilage matrix proteins by BMP-transfected murine mesenchymal stem cells encapsulated in a novel class of alginates. Biomaterials 23(9):2003-2013

Wolters GHJ, Fritschy WM, Gerrits D, van Schilfgaarde R (1992) A versatile alginate droplet generator applicable for microencapsulation of pancreatic islets. J Appl Biomater 3(4):281-286. doi:10.1002/jab.770030407

Worthington AM (1908) A study of splashes. Longmans, Green, and Co., London

Xu L, Zhang WW, Nagel SR (2005) Drop splashing on a dry smooth surface. Phys Rev Lett 94(18):184505

Zhang W, Yang G, Zhang A, Xu LX, He X (2009) Preferential vitrification of water in small alginate microcapsules significantly augments cell cryopreservation by vitrification. Biomed Microdevices 12(1):89-96. doi:10.1007/s10544-009-9363-z

Zimmermann U, Thürmer F, Jork A, Weber M, Mimietz S, Hillgartner M, Brunnenmeier F, Zimmermann H, Westphal I, Fuhr G, Noth U, Haase A, Steinert A, Hendrich C (2001) A novel class of amitogenic alginate microcapsules for long-term immunoisolated transplantation. Ann N Y Acad Sci 944:199-215. doi:10.1111/j.1749-6632.2001.tb03833.x

Zimmermann H, Hillgartner M, Manz B, Feilen P, Brunnenmeier F, Leinfelder U, Weber M, Cramer H, Schneider S, Hendrich C, Volke F, Zimmermann U (2003) Fabrication of homogeneously crosslinked, functional alginate microcapsules validated by NMR-, CLSM- and AFM-imaging. Biomaterials 24(12):2083-2096 pii:S0142961202006397

Zimmermann H, Zimmermann D, Reuss R, Feilen PJ, Manz B, Katsen A, Weber M, Ihmig FR, Ehrhart F, Gessner P, Behringer M, Steinbach A, Wegner LH, Sukhorukov VL, Vasquez JA, Schneider S, Weber MM, Volke F, Wolf R, Zimmermann U (2005) Towards a medically approved technology for alginatebased microcapsules allowing long-term immunoisolated transplantation. J Mater Sci Mater Med 16(6):491-501. doi:10.1007/ s10856-005-0523-2

Zimmermann H, Ehrhart F, Zimmermann D, Müller D, Katsen-Globa A, Behringer M, Feilen PJ, Gessner P, Zimmermann G, Shirley SG, Weber MM, Metze J, Zimmermann U (2007a) Hydrogelbased encapsulation of biological, functional tissue: fundamentals, technologies and applications. Appl Phys A 89:909-922. doi:10.1007/s00339-007-4270-8

Zimmermann H, Shirley SG, Zimmermann U (2007b) Alginate-based encapsulation of cells: past, present, and future. Curr Diab Rep 7(4):314-320

Zimmermann U, Cramer H, Jork A, Thürmer F, Zimmermann H, Fuhr G, Hasse C, Rothmund M (2008) Microencapsulation-based cell therapy. In: Rehm H-J, Reed G (eds) Biotechnology: special processes, vol 10, 2nd edn. Wiley-VCH Verlag GmbH, Weinheim, Germany. doi:10.1002/9783527620937.ch19 